\title{
EFFECTS OF THE ROTATIONAL VEHICLE DYNAMICS ON THE ASCENT FLIGHT TRAJECTORY OF THE SPACELINER CONCEPT
}

\author{
Sven Krummen ${ }^{(1)}$, Martin Sippel ${ }^{(1)}$ \\ ${ }^{(1)}$ German Aerospace Center (DLR), Institute of Space Systems, Space Launcher System Analysis, \\ Robert-Hooke-Straße 7, 28359 Bremen, Germany \\ sven.krummen@dlr.de, martin.sippel@dlr.de
}

\begin{abstract}
During the preliminary design of space transportation systems the vehicle dynamics are commonly reduced to a pointmass model for definition of the flight trajectory. While this approach effectively reduces the number of model parameters in the design process, it neglects the rotational dynamics of the vessel completely. Since the rotational degrees of freedom (DOF) have a significant influence on the vehicle's controllability, a sole analysis of the translational dynamics is insufficient to assess the general feasibility of the concept.

This study investigates the ascent flight trajectory of the SpaceLiner vehicle, a concept for a hypersonic suborbital space plane, based on a newly developed 6-DOF flight dynamics simulation to determine the influence of the rotational dynamics on the vehicle's controllability and performance. The first part of this paper will focus on the developed vehicle model which features a transient inertia model as well as an algorithmic-designed flight control system. The second part will present several simulations of nominal and off-nominal ascent trajectories. Based on the results it will be shown that SpaceLiner's thrust vector control system is sufficiently dimensioned for the investigated mission scenarios, while the vehicle performance is only slightly influenced by the rotational dynamics.
\end{abstract}

\section{Keywords}

Launcher, Spaceplane, Flight Dynamics, Trajectory Simulation, Thrust Vector Control

\section{INTRODUCTION}

The SpaceLiner is a concept of a hypersonic suborbital launch vehicle, which is designed to be capable of transporting 50 passengers over ultra-long-haul distances. Since 2005 this concept has been researched by the German Aerospace Center (DLR) [1], leading to the investigation of various different vehicle configurations [2].

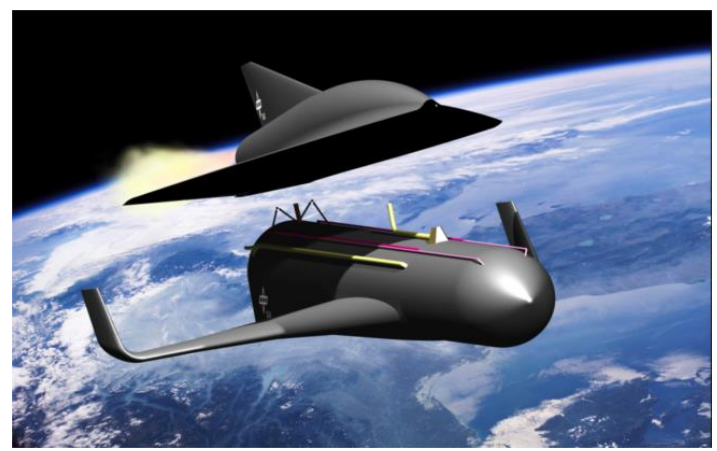

FIG 1. Artist's impression of the SpaceLiner 7-3 during stage separation

The currently proposed baseline design, also known as SpaceLiner 7-3, is shown in FIG 1. As can be seen the vehicle consists of two mated stages: the passenger stage
(SLP) and a booster stage (SLB) [3]. Both stages are propelled by 11 bipropellant LOX/LH2 rocket engines (SLME), possessing 9 engines on the SLB and 2 on the SLP stage [4]. The cryogenic propellants for all engines are stored inside the fuselage of both stages, also allowing for a cross-feed from the SLB tanks to the SLP engines [5]. All passengers are seated in the SpaceLiner Passenger Capsule (SLC) in the forward compartment of the SLP stage which could be ejected in case of hazardous flight anomalies [6]. An overview of the vehicle's system architecture is depicted in FIG 2.

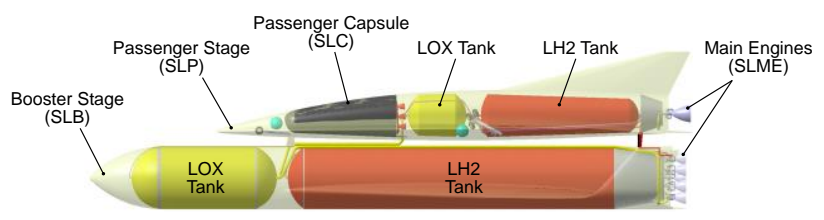

FIG 2. Overview of the SpaceLiner's major subsystems

During operation the SpaceLiner Vehicle (SLV) lifts off vertically in mated configuration and climbs to an altitude of approximately $80 \mathrm{~km}$. After stage separation the reusable booster stage returns to the launch site while the passenger stage continues accelerating to a flight-path velocity of approximately $7.2 \mathrm{~km} / \mathrm{s}$ before its main 
engines are turned off. Henceforth the passenger stage performs a continuous gliding reentry flight to its destination, capable of covering a ground range of up to $17000 \mathrm{~km} \mathrm{[7].} \mathrm{This} \mathrm{distance} \mathrm{corresponds} \mathrm{to} \mathrm{the} \mathrm{typical}$ reference mission from Australia to Europe as visualized in FIG 3, which can be served by the SpaceLiner in less than 2 hours.

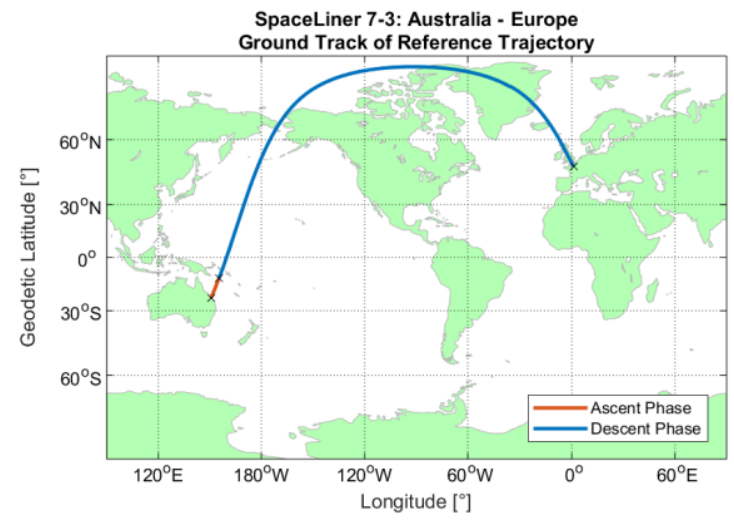

FIG 3. Ground track of the SpaceLiner reference mission from Australia to Europe

In previous system engineering studies the vehicle subsystems [8] and feasible flight trajectories [9] have already been specified. However, in these investigations the vehicle dynamics have been idealized to a point mass model, considering only the translational vehicle movement of the vehicle. A new study has now been conducted in order to assess the influence of the rotational degrees of freedom on the concept's feasibility [10]. This investigation is based on a flight dynamics simulation capable of determining the vehicle's state of motion in six degrees of freedom (DOF) and a compatible SpaceLiner Vehicle model. Since the SpaceLiner can be seen as a typical example for any non-symmetrical launcher concept, the applied methods can easily be extended to similar vehicle configurations.

\section{VEHICLE MODELING}

For the investigation of the SpaceLiner's ascent flight dynamics a vehicle model has been developed, which extends the level of detail of previous system definitions [3]. This model is implemented in the TRACE Simulation Framework, a DLR-internal tool based on Matlab/Simulink [11] for simulating vehicle dynamics with up to 6 degrees of freedom. The top-level structure of this simulation is sketched in FIG 4.

The system's model can be divided into two general sections: The SpaceLiner Vehicle Model, which describes the transient vehicle properties, and the Flight Control System, which provides a preliminary feed-back loop for the thrust vector control system (TVC).

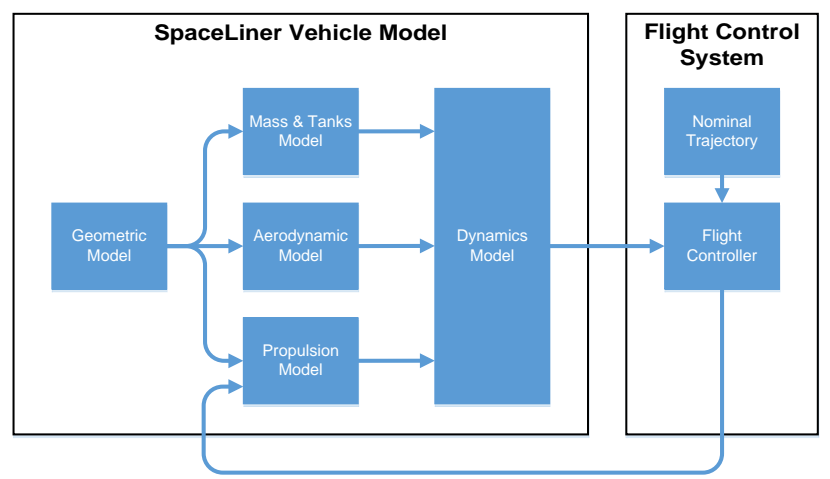

FIG 4. Top-level structure of the SpaceLiner flight dynamics simulation

\subsection{SpaceLiner Vehicle Model}

The SpaceLiner Vehicle Model has been derived from the most recent vehicle specification as sketched in FIG 5 and FIG 6. Its purpose is to determine the vehicle's mass and inertia properties in every flight state, as well as the resulting aerodynamic and propulsive forces on the vehicle. Since these properties are subject to large variations during the ascent flight, the non-linear and time-dependent effects needed to be considered explicitly by domain-specific submodels.
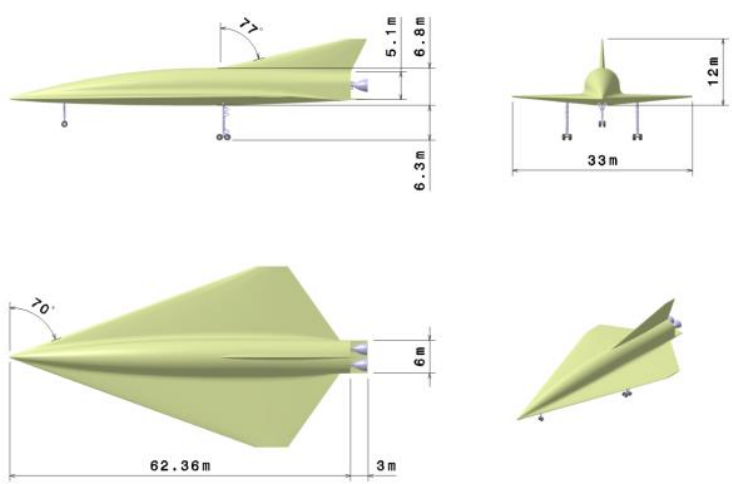

FIG 5. Main dimensions of the SLP Stage
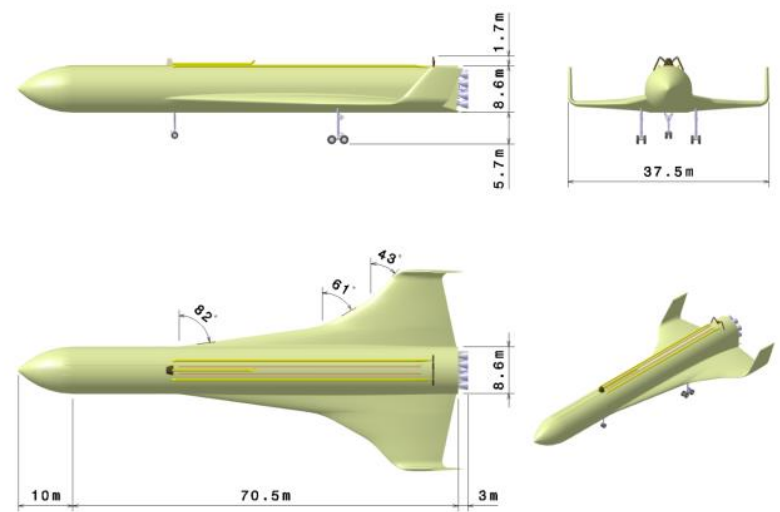

FIG 6. Main dimensions of the SLB Stage 


\subsubsection{Inertia Model}

For the flight dynamics simulation, the SpaceLiner vehicle has been modeled as a rigid body with a time-varying mass and inertia tensor. Basically, two distinct factors are contributing to the vehicle's inertia properties during ascent flight: The vast majority of the vehicle's inertia at lift-off is caused by the loaded propellant mass, while a constant minority arises from the structure and subsystems of the vehicle.

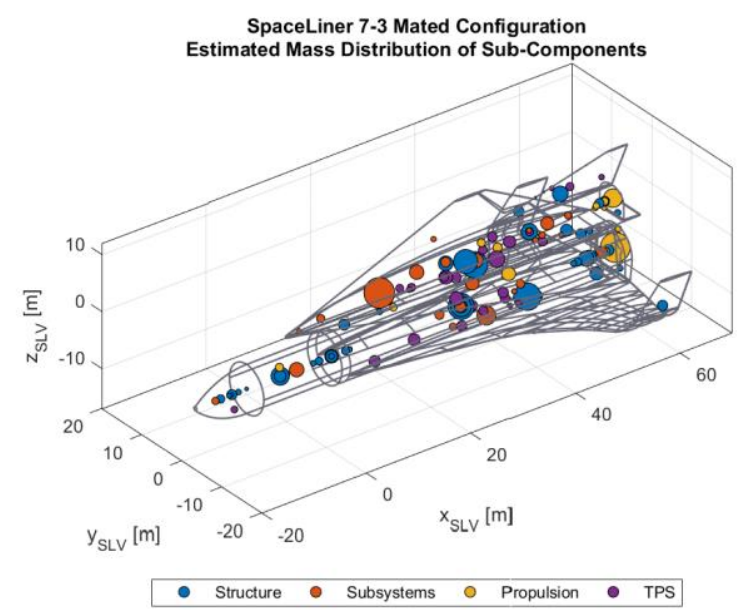

FIG 7. Mass model of the SpaceLiner subsystems; the volume of each sphere is proportional to the corresponding subsystem mass

As visualized in FIG 7 the dry mass of the SpaceLiner has been specified on a subsystems level. The corresponding mass and inertia properties of each component have already been estimated by previous system specifications [12]. A summary of the vehicle mass breakdown is provided in TAB 1 . Within the scope of this study it is assumed that any displacement of mass components due to structural-elastic effects or actuator movements has only a secondary impact on the ascent flight dynamics. Therefore all dry masses have been considered with a constant location and orientation within the vehicle-fixed frame.

TAB 1. Major mass groups of the SpaceLiner Vehicle

\begin{tabular}{llll}
\hline Mass Group & SLB Stage & SLP Stage & Mated SLV \\
\hline Structure & $124.6 \mathrm{t}$ & $56.3 \mathrm{t}$ & $180.9 \mathrm{t}$ \\
Subsystems & $20.1 \mathrm{t}$ & $46.5 \mathrm{t}$ & $66.6 \mathrm{t}$ \\
Propulsion & $37.3 \mathrm{t}$ & $10.6 \mathrm{t}$ & $47.9 \mathrm{t}$ \\
TPS & $19.1 \mathrm{t}$ & $26.6 \mathrm{t}$ & $45.7 \mathrm{t}$ \\
\hline Total Dry & $201 \mathrm{t}$ & $140 \mathrm{t}$ & $341 \mathrm{t}$ \\
Propellant & $1284 \mathrm{t}$ & $230 \mathrm{t}$ & $1514 \mathrm{t}$ \\
\hline GLOW & $1485 \mathrm{t}$ & $370 \mathrm{t}$ & $1855 \mathrm{t}$ \\
\hline
\end{tabular}

In order to determine the variable propellant mass distribution inside the vehicle a simplified volume model of the propellant system has been established. As shown in FIG 8 this model has been derived from the SpaceLiner's tanks and feedline geometry [5]. During simulation the filling level of each tank and feedline is calculated dynamically to determine the instantaneous inertia tensor of the vehicle. For the propellant allocation inside the tanks a frozen fluid model with a hydrostatic draining cascade of the tanks and feedlines from fore to aft has been utilized. Although this model simplifies the thermo- and fluid dynamic effects during the draining process it can be treated as a valid approximation since the SpaceLiner is subject to a continuous axial acceleration in the range of $1.3-2.5 \mathrm{~g}$ during ascent flight which allocates most propellant mass in the rear volume of the tanks. As the vehicle does not encounter any microgravity environment before Main Engine Cut-Off (MECO) propellant sloshing can only take place at the free surface towards the ullage volume. Due to the slender geometry of the tanks only a minor mass fraction of the propellant can be incorporated in this dynamic process. Because this fluid motion could also be damped further by technical devices inside the tanks, fuel sloshing has been neglected within the scope of this study.

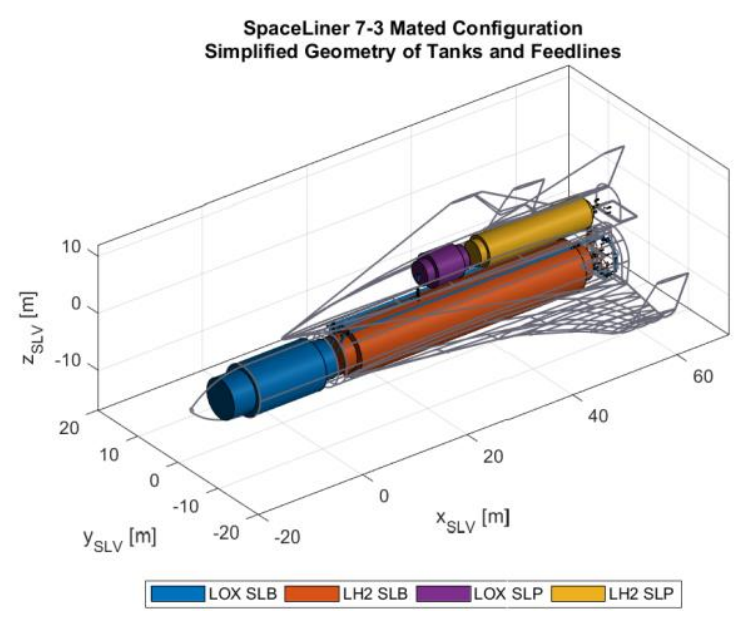

FIG 8. Simplified volume model of the SpaceLiner's propellant system

Further analyses of the time-varying inertia properties of the SpaceLiner Vehicle during ascent flight will be presented in chapter 4.1 .

\subsubsection{Aerodynamics Model}

In addition to the inertia properties the aerodynamic characteristics of the SpaceLiner also change significantly during ascent flight as the vehicle passes through sub-, super- and hypersonic flow regimes with aerodynamic pressures between $0.1-30 \mathrm{kPa}$. In previous studies an aerodynamic reference database for the SpaceLiner has been defined which determines the longitudinal aerodynamic coefficients for multiple flight conditions based on CFD calculations and empirical methods [13]. For the aerodynamic model of the SpaceLiner this dataset has been complemented by estimations of lateral and dynamic derivatives of the aerodynamic coefficients [14]. All coefficients and derivatives have been considered with respect to the Mach regime and the vehicle's angle of attack as it is visualized in FIG 9 for the lift-to-drag ratio. 


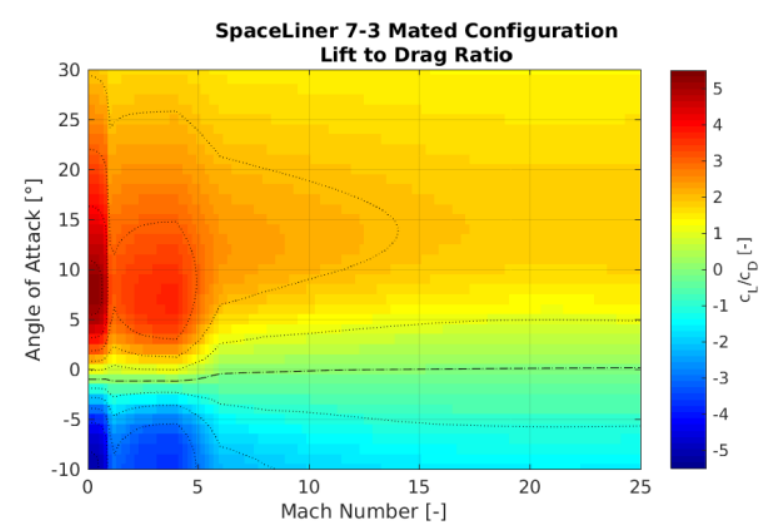

FIG 9. Contour plot of the lift-to-drag ratio of the mated SpaceLiner vehicle for different flight conditions

Investigations of the aerodynamic stability and trimmability of the SpaceLiner will be presented in chapter 4.4 .

\subsubsection{Propulsion Model}

During ascent flight the SpaceLiner is propelled by the SpaceLiner Main Engines (SLME) with 2 engines attached to the SLP stage and 9 engines attached to the SLB stage. While all engines employ the same full-flow staged combustion cycle, the nozzle extension of the SLP engines has a larger expansion ratio for optimized performance in higher altitudes. Furthermore, all SLMEs are designed to be throttleable by reducing the oxidizer mass flow during operation. The engines characteristics for these operating points have already been determined in previous simulation studies of the engine cycle [4]. A summary of the results is provided in TAB 2.

TAB 2. Characteristics of the SpaceLiner Main Engines

\begin{tabular}{lllll}
\hline Engine Data & \multicolumn{2}{l}{ SLME (SLB) } & \multicolumn{2}{l}{ SLME (SLP) } \\
\hline Propellant & LOX / LH2 & \multicolumn{2}{l}{ LOX / LH2 } \\
Mixture Ratio [-] & 5.5 & 6.5 & 5.5 & 6.5 \\
Pressure MCC [MPa] & 15.1 & 16.9 & 15.1 & 16.9 \\
Expansion Ratio [-] & 33 & 33 & 59 & 59 \\
\hline Spec. Impulse (vac) [s] & 439 & 435 & 451 & 448 \\
Spec. Impulse (msl) [s] & 387 & 390 & 357 & 367 \\
\hline Thrust (vac) [kN] & 2061 & 2356 & 2116 & 2425 \\
Thrust (msl) [kN] & 1817 & 2111 & 1678 & 1986 \\
\hline
\end{tabular}

In order to provide thrust vector control (TVC) during ascent flight each engine is designed to gimbal independently around its idle position. A mechanical gimbal limit of $\pm 8^{\circ}$ has been specified for all engines. During simulation the necessary engine deflections are determined by the flight control system.

The feasibility of the specified TVC system will be assessed in chapter 4.3 , while the vehicle's trimmability will be analyzed in chapter 4.4 .

\subsection{Flight Control System}

Additionally to the SpaceLiner Vehicle Model a preliminary flight control system has been designed to operate the TVC actuators during ascent flight. In previous investigations analyzing the translational motion of the SpaceLiner only, a simple feed-forward controller in combination with an offline optimization algorithm has been utilized [15]. However, for a 6 DOF trajectory simulation this approach is not applicable since the state variables are strongly coupled and sensitive to changes in the control variables. This issue is solved by implementing a closed-loop controller which determines the control variables at simulation runtime.

A block diagram representing the top-level structure of the implemented flight control system is shown in FIG 10. As can be seen the control system adapts the classical cascade design of flight controllers for airplanes [16]. Here, the inner feedback loop is controlling the vehicle's attitude, while the outer feedback loop is providing flight-path control. The target states of the vehicle, which are derived from a reference trajectory, are commanded to the controller.

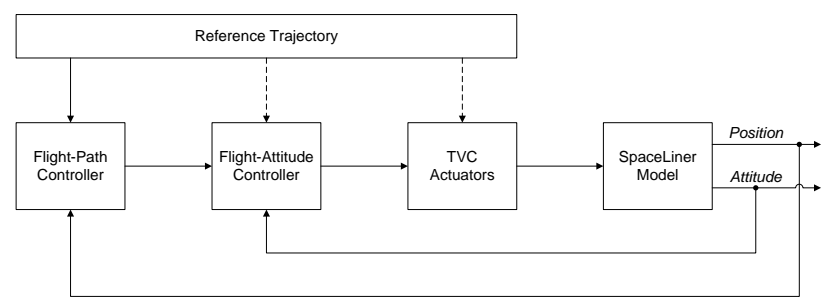

FIG 10. Cascade structure of the implemented flight control system

On the lowest level the individual deflections of each SLME are combined to 3 control signals for attitude control which are controlled by PID feedback controllers. In particular, for pitch control all engines are deflected simultaneously in vertical direction, while yaw control is provided by equivalent lateral deflections. Roll control is executed during mated ascent flight by inducing an additional horizontal deflection of the passenger stage engines only since they have the largest lever to the vehicle's center of gravity. After stage separation roll control is realized by opposed vertical deflections of these two engines. In the current setup additional attitude control by the aerodynamic control surfaces or the reaction control system (RCS) is not intended as the moments generated by the TVC system have the highest control authority during ascent flight.

The inner feedback loop for attitude control is enclosed by the flight-path control loop which controls the translational deviations between a commanded trajectory and the actual position of the SpaceLiner. This control loop includes two PID controllers for vertical pitch and lateral skid-to-turn maneuvers. A lateral bank-to-turn and a throttling controller is also integrated on this level but 
currently not used.

In order to counteract the non-linear and transient system behavior of the SpaceLiner vehicle dynamics, the feedback gains of all PID controllers are provided by a gain-scheduling scheme based on the particular flight condition. Additionally, the trim settings for each operating point are also determined and fed forward to the TVC system to improve the follow-up behavior of the controller.

For each operating point the feedback gains are designed by a semi-automatic relay autotuning algorithm [17][18]. In this procedure the PID controllers are individually replaced by relay elements during simulation, which are inducing a continuous oscillation of the system. Based on resulting oscillation amplitude and frequency the ultimate gain $\mathrm{K}_{\mathrm{u}}$ and frequency $\omega_{\mathrm{u}}$ of the system can be identified. These system characteristics are used to determine adequate feedback gains by use of the empirical ZieglerNichols tuning rules [19].
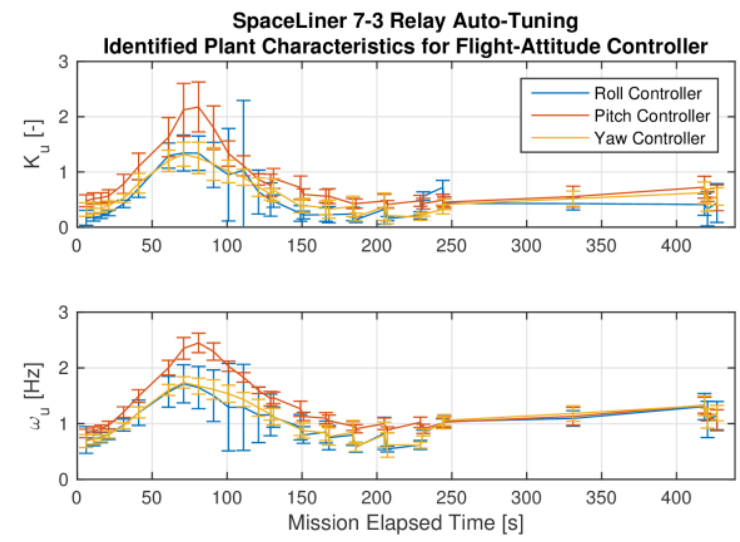

FIG 11. System characteristics of the SpaceLiner attitude control loop as identified by the relay autotuning algorithm

The identified system characteristics of the SpaceLiner's attitude control loop are shown in FIG 11. As can be seen the system parameters show a significant measuring spread for the operating points between $\mathrm{t}_{\mathrm{MET}}=60 \mathrm{~s}$ and $\mathrm{t}_{\mathrm{MET}}=130 \mathrm{~s}$ which is caused by some outliers in the measurements due to the empirical approach of this design method. Despite these inaccuracies the resulting feedback gains for the flight controller revealed sufficient quality during simulation, as will be shown in the following chapters.

Generally, in the current preliminary design phase of the SpaceLiner concept a semi-automatic control design procedure is preferable in order to adapt the flight control system fast to any changes in the vehicle specifications. Therefore further rapid control prototyping algorithms are currently under investigation and will be compared against the relay autotuning procedure in a future study.

\subsection{Dynamics and Environment Model}

The vehicle's equations of motion over the globe have been implemented by a separate dynamics model which is non-specific for this vehicle. For the trajectory calculation the inertial position and attitude matrix have been integrated numerically [20], while all motions have been referred to the elliptical datum of the World Geodetic System (WGS84) [21].

The gravitational environment has also been modeled as an elliptical potential based on the Earth Gravitational Model (EGM96) up to degree 2 [21]. The undisturbed atmosphere model consists of the definitions of the U.S. Standard Atmosphere (US76) [22]. In case of ascent flight simulations under atmospheric disturbances this model can be extended by the Horizontal Wind Model (HWM93) [23] and the Von Kármán Wind Turbulence Model (Karman) [24]. For both models light, moderate and severe disturbance intensities have been defined.

\section{MISSION SCENARIOS}

To determine the influence of the rotational DOFs on the SpaceLiner's ascent flight dynamics a simulation study has been concluded investigating a nominal and several off-nominal ascent trajectories. For this study the reference mission from Australia to Europe has been chosen as the base scenario. The major flight events during this mission are visualized in FIG 12. It should be noticed that the SpaceLiner Main Engines are throttled and sequentially cut off in upper atmospheric layers to limit the acceleration on the passengers to $2.5 \mathrm{~g}$ [25]. These events are of particular interest for the controllability of the vehicle since the engine cut-offs cause an asymmetric thrust setting during periods of the ascent flight.

The ascent trajectory of the SpaceLiner on this reference mission has been simulated with and without atmospheric disturbances to identify possible sensitivities in the vehicle dynamics. Within the scope of this study the influence of global average wind profiles (HWM93) and local stochastic gusts (Karman) have been investigated, both limited to moderate intensities only.

Furthermore several operational anomaly scenarios have been considered in the study which might be critical to the mission's success. Previous studies have already identified a reduced specific impulse of the SLMEs due to imperfect combustion as a potential anomaly scenario, as well as premature stage separation [26]. Additionally to these scenarios isolated engine failures at Lift-Off and immediately before Max-Q have been simulated. In both cases a failure of the outermost engine on the SLB stage has been investigated since this scenario would generate the largest moments on the vehicle. 


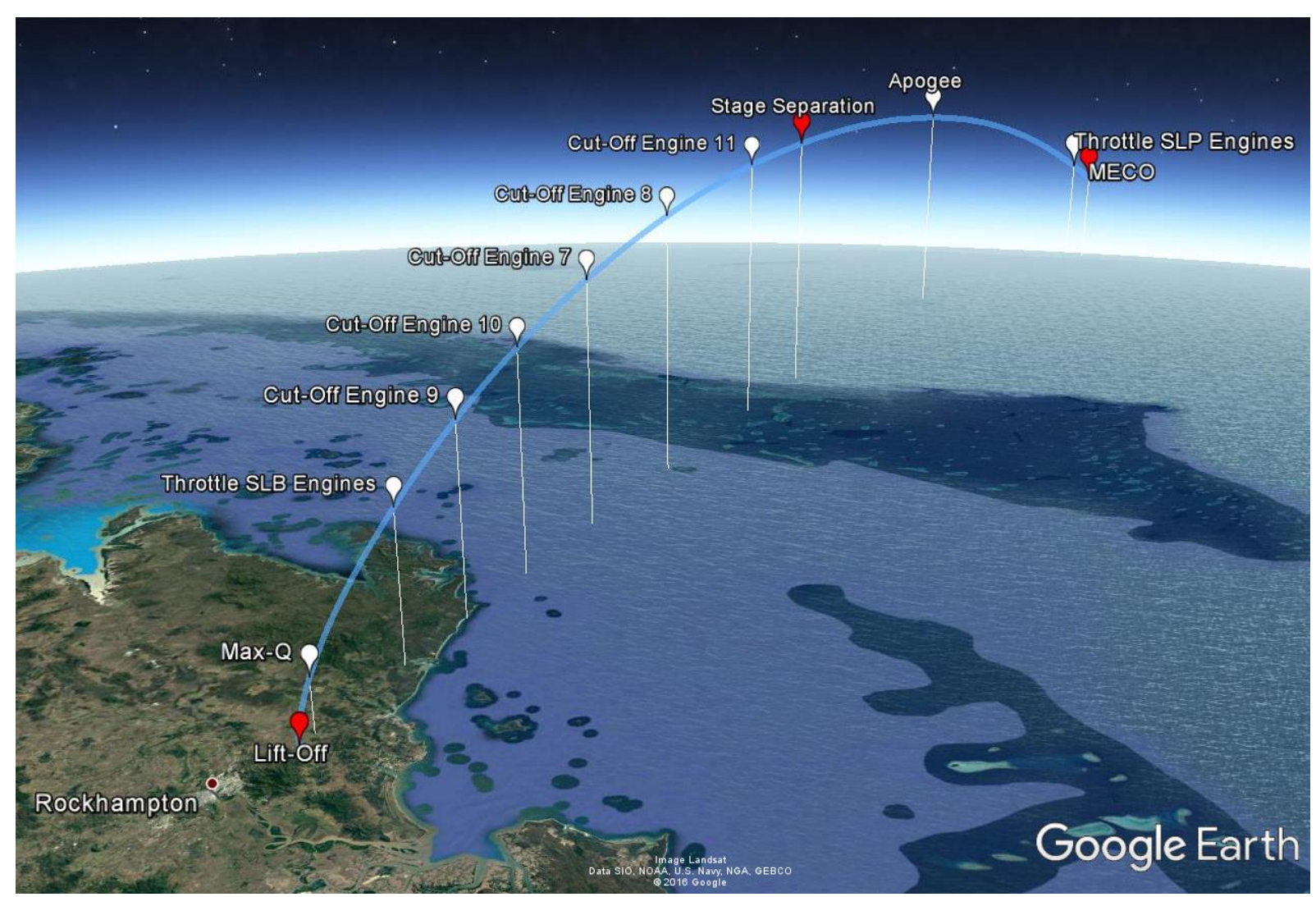

FIG 12. Nominal ascent trajectory of the SpaceLiner reference mission from Australia to Europe displaying all major mission events

An engine failure on the SLP stage could not be analyzed so far as in this case roll control needs to be provided by the onboard reaction control system (RCS) after the stage separation. A typically pulsed RCS controller is currently not compatible with the architecture of the preliminary flight control system and will be considered in future studies.

In summary the following simulation cases have been investigated:

1) Nominal undisturbed ascent trajectory

2) Ascent trajectories with atmospheric disturbances
a) Large scale wind profile (HWM93)
b) Moderate stochastic gusts (Karman)
c) Combined disturbances (HWM93 \& Karman)

3) Ascent trajectories under anomaly scenarios
a) Reduced $\mathrm{I}_{\mathrm{sp}}$ of the booster's SLMEs by $-3 \mathrm{~s}$
b) Premature stage separation by $-6.5 \mathrm{~s}$
c) Failure of outermost SLME at Lift-Off
d) Failure of outermost SLME at Max-Q

The objective for all simulation cases is to follow a predefined reference trajectory. For cases 1) and 2a-c) the nominal ascent path has been chosen and commanded to the flight control system, while for cases 3a-d) respectively adapted non-nominal trajectories have been utilized. These reference trajectories were previously designed and optimized based on a 3 DOF model [15].

\section{SIMULATION RESULTS AND ANALYSIS}

The simulation results for all defined mission scenarios will be presented in the following paragraphs. First the transient vehicle properties of the SpaceLiner will be analyzed, before the simulated ascent trajectories will be compared against their reference trajectories in terms of accuracy and performance. Based on these results the feasibility of the TVC system will be assessed. Finally, the controllability of the SpaceLiner vehicle during ascent flight will be investigated in more detail.

\subsection{Evolution of the Vehicle Properties}

As typical for all space transportation systems, the mass and inertia properties of the SpaceLiner depend strongly on the current filling level of the propellant tanks. Specifically for the SpaceLiner Vehicle the loaded propellant at Lift-Off contributes to $82 \%$ of the vehicle's wet mass and forms over $99 \%$ of its principle moments of inertia. The changes in these properties during ascent flight are monitored by the inertia model of the trajectory simulation.

The movement of the vehicle's center of gravity (COG) during ascent flight, which is caused by the continuous draining of the propellant tanks, is visualized in FIG 13. During the mated flight phase of both SpaceLiner stages, the COG is shifting $21.7 \mathrm{~m}$ backwards and $3.4 \mathrm{~m}$ upwards, 
which corresponds to $27 \%$ of the vehicle's length and $15 \%$ of the respective height. Since this movement is significant compared to the vehicle's dimension the trim settings of the TVC system need to be adjusted in all flight states. This holds particularly true as the positions of the resulting center of thrust (COT) and center of aerodynamic pressure (COP) are also subject to changes during ascent flight. While the evolution of the COP is caused by the different aerodynamic flow regimes and flight attitudes, the movement of the COT arises from the sequential, asymmetric throttling of the SLMEs.

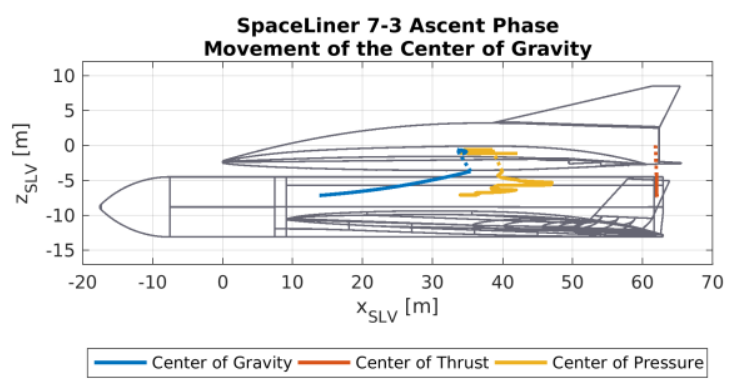

FIG 13. Transient movement of the SpaceLiner's center of gravity, center of thrust and center of aerodynamic pressure during nominal ascent flight

In addition to the varying introduction points of the aerodynamic and thrust force vectors, the total magnitude of external forces also change during ascent flight. FIG 14 displays the relations of the external forces acting on the vehicle. It can be seen that the thrust force is dominant in all flight phases, while the vehicle's aerodynamics only have a considerable influence for the first $150 \mathrm{~s}$ of the ascent flight. The point of maximum aerodynamic load (Max-Q) can be identified at $\mathrm{t}_{\mathrm{MET}} \approx 75 \mathrm{~s}$.

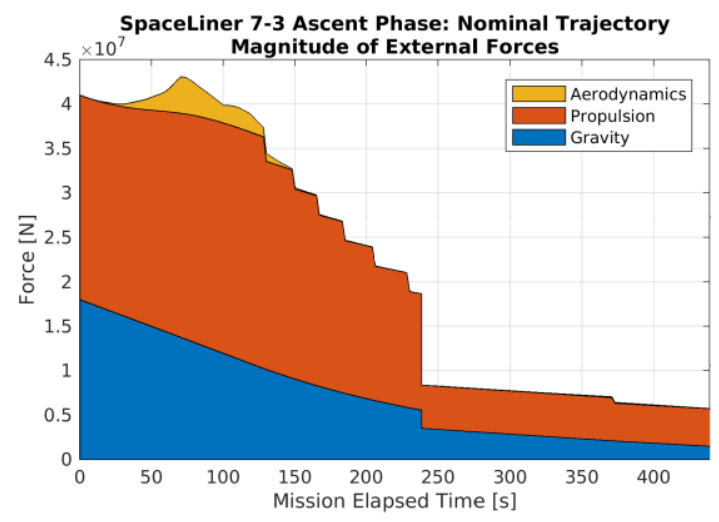

FIG 14. Magnitude of the external forces acting on the SpaceLiner vehicle during nominal ascent flight

\subsection{Deviations from the Reference Trajectories}

The influence of the rotational degrees of freedom on the SpaceLiner's ascent trajectory can be estimated by comparing the simulated trajectories with the commanded reference values, since these reference trajectories have been determined by pure translational calculations. In this comparison two possible influences are particularly interesting: reductions in the vehicle's positioning accuracy while following the commanded trajectory and additional performance losses due to the rotational movement of the vessel.

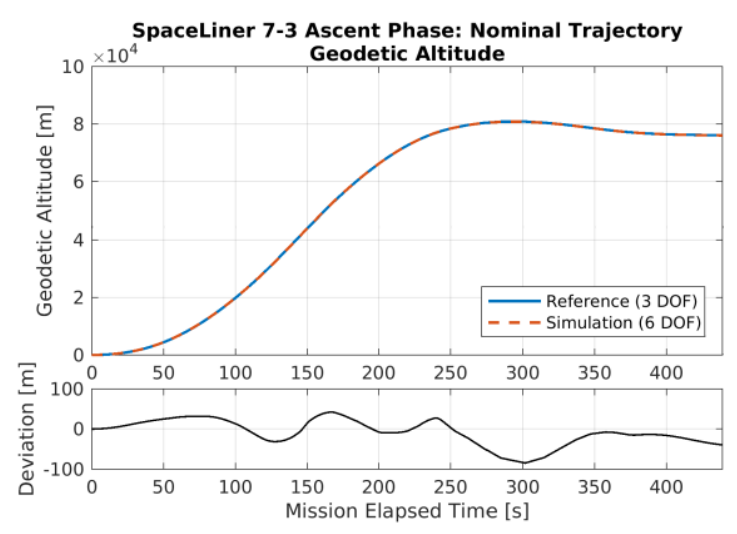

FIG 15. Simulation results for the nominal ascent trajectory; comparison of the simulated and commanded vehicle altitude

The deviation between the commanded and simulated altitude of the SpaceLiner vehicle for the undisturbed ascent flight is shown in FIG 15. It can be seen that the maximum vertical displacement of the SpaceLiner with respect to the reference trajectory remains below $85 \mathrm{~m}$ in any flight condition while no continuous drift can be identified. Similar deviations can be observed for all simulation cases as summarized in TAB 3. Generally, a positioning accuracy below $100 \mathrm{~m}$ can be achieved in vertical direction and below $60 \mathrm{~m}$ in lateral direction. For most flight phases these accuracies can be considered as non-critical as they are in the same order of magnitude as the vehicle's dimensions.

TAB 3. Maximum vertical and lateral displacements of the SpaceLiner vehicle from the commanded reference trajectory

\begin{tabular}{llll}
\hline Simulation Case & $\begin{array}{l}\text { Max. Vertical } \\
\text { Error }\end{array}$ & $\begin{array}{l}\text { Max. Lateral } \\
\text { Deviation }\end{array}$ \\
\hline 1) & Nominal Ascent & $84.673 \mathrm{~m}$ & $56.920 \mathrm{~m}$ \\
\hline 2a) & HWM93 & $85.214 \mathrm{~m}$ & $59.118 \mathrm{~m}$ \\
2b) & Karman & $84.767 \mathrm{~m}$ & $56.922 \mathrm{~m}$ \\
2c) & HWM93 \& & $85.269 \mathrm{~m}$ & $59.015 \mathrm{~m}$ \\
& Karman & & \\
\hline 3a) & Reduced Isp & $92.705 \mathrm{~m}$ & $55.591 \mathrm{~m}$ \\
3b) & Separation Time & $86.761 \mathrm{~m}$ & $56.909 \mathrm{~m}$ \\
3c) & Failure Lift-Off & $60.767 \mathrm{~m}$ & $52.977 \mathrm{~m}$ \\
3d) & Failure Max-Q & $79.396 \mathrm{~m}$ & $59.187 \mathrm{~m}$ \\
\hline
\end{tabular}

The strictest constraint to the positioning accuracy occurs during take-off since a collision with the Launchpad Tower needs to be avoided. For the considered simulation cases the flight-paths during the vertical lift-off phase are shown in FIG 16. During this first period a maximum deviation of $3.0 \mathrm{~m}$ from the reference path can be determined for ascent under atmospheric disturbances, as well as a deviation of $4.5 \mathrm{~m}$ in case of the most critical 
engine failure at lift-off. These margins need to be considered in the design of the launch pad.

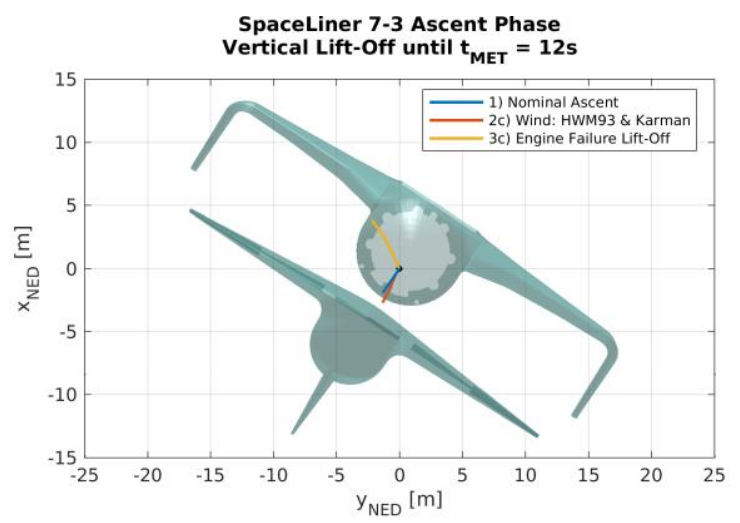

FIG 16. Flight-path deviations during the vertical lift-off phase for each simulation case

Regarding the rotational dynamics of the SpaceLiner, FIG 17 visualizes the difference of the simulated angle of attack to its reference values. Here, the regular angle of attack with respect to the body-fixed frame $\alpha_{\mathrm{SLV}}$, as well as the angle of attack of the resulting thrust vector $\alpha_{\mathrm{SLME}}$, is displayed. For the first flight phases it can be observed that the reference values are tracked by $\alpha_{\mathrm{SLV}}$ sufficiently, while in higher altitudes they are followed by $\alpha_{\mathrm{SLME}}$. This artifact is caused by the changing moment trimming mechanism in different atmospheric layers. In denser layers the aerodynamic moment needs to be trimmed by the TVC system, whereas the thrust-induced moment is the dominant force in upper layers. A similar behavior can be observed for the sideslip angle. In general it can be stated that no corrective maneuvers with excessive aerodynamic angles are executed in any simulation case, even during the sequential, asymmetric cut-off of the SpaceLiner Main Engines.

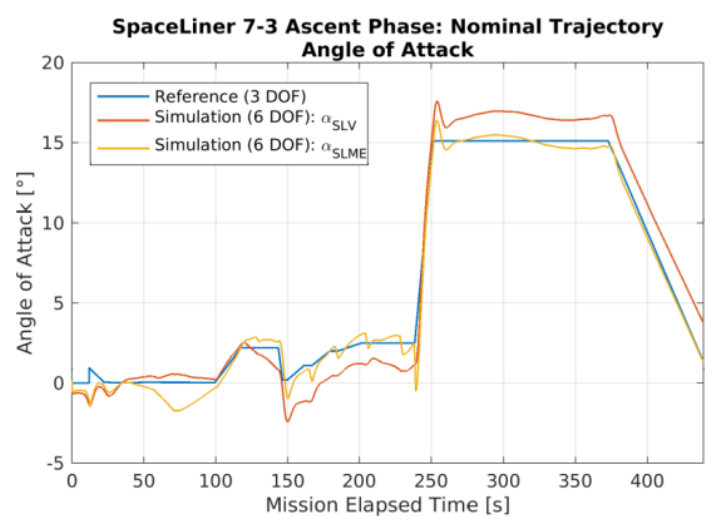

FIG 17. Comparison of the simulated and commanded angle of attack for the nominal ascent trajectory

To estimate the influence of the rotational degrees of freedom on the flight performance a comparison of the vehicle's state of motion at Main Engine Cut-Off (MECO) is given in TAB 4. For all simulation cases the final velocity of the SpaceLiner is reduced between $\Delta \mathrm{v} \approx 4 \mathrm{~m} / \mathrm{s}$ to $\Delta \mathrm{v} \approx 11 \mathrm{~m} / \mathrm{s}$ compared to the reference trajectories. However, since in all cases the vehicle's altitude at MECO is also slightly reduced, a sole quantification of the performance loss in terms of Delta- $\mathrm{V}$ is not sufficient. A more convenient performance measure is the specific orbital energy which considers the kinetic as well as the potential energy of the vehicle. For the disturbed and undisturbed ascent scenarios a relative performance loss of approximately $0.1 \%$ compared to the reference trajectory can be observed, while a relative loss of up to $0.3 \%$ occurs in the anomaly scenarios. Losses in this order of magnitude are covered by the preliminary design margins of the SpaceLiner vehicle.

TAB 4. Simulated flight state at Main Engine Cut-Off (MECO) for each simulation case with absolute and relative errors to the commanded reference trajectory

\begin{tabular}{|c|c|c|c|c|}
\hline \multicolumn{2}{|c|}{ Simulation Case } & \multirow{2}{*}{$\begin{array}{l}\text { Altitude (MECO) } \\
\mathbf{7 6 . 0 4 7} \mathbf{~ k m} \\
(-40.450 \mathrm{~m} ;-0.053 \%)\end{array}$} & \multirow{2}{*}{$\begin{array}{l}\text { Velocity }(\text { MECO) } \\
7.299 \mathbf{~ k m} / \mathbf{s} \\
(-3.958 \mathrm{~m} / \mathrm{s} ;-0.054 \%)\end{array}$} & \multirow{2}{*}{$\begin{array}{l}\text { Spec. Energy (MECO) } \\
-3.382 \mathbf{e}^{2} \mathbf{~ m}^{2} / \mathbf{s}^{2} \\
\left(-22127 \mathrm{~m}^{2} / \mathrm{s}^{2} ;-0.077 \%\right)\end{array}$} \\
\hline 1) & Nominal Ascent & & & \\
\hline 2a) & $\begin{array}{l}\text { Disturbed Ascent: } \\
\text { HWM93 }\end{array}$ & $\begin{array}{l}\mathbf{7 6 . 0 4 7} \mathbf{~ k m} \\
(-40.398 \mathrm{~m} ;-0.053 \%)\end{array}$ & $\begin{array}{l}7.299 \mathbf{~ k m} / \mathbf{s} \\
(-4.057 \mathrm{~m} / \mathrm{s} ;-0.056 \%)\end{array}$ & 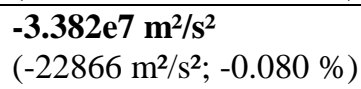 \\
\hline 2b) & $\begin{array}{l}\text { Disturbed Ascent: } \\
\text { Karman }\end{array}$ & $\begin{array}{l}76.047 \mathbf{~ k m} \\
(-40.128 \mathrm{~m} ;-0.053 \%)\end{array}$ & $\begin{array}{l}7.299 \mathrm{~km} / \mathrm{s} \\
(-4.805 \mathrm{~m} / \mathrm{s} ;-0.066 \%)\end{array}$ & 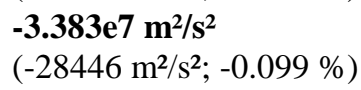 \\
\hline 2c) & $\begin{array}{l}\text { Disturbed Ascent: } \\
\text { HWM93 \& Karman }\end{array}$ & $\begin{array}{l}76.047 \text { km } \\
(-40.075 \mathrm{~m} ;-0.053 \%)\end{array}$ & $\begin{array}{l}7.298 \mathbf{~ k m} / \mathbf{s} \\
(-4.901 \mathrm{~m} / \mathrm{s} ;-0.067 \%)\end{array}$ & 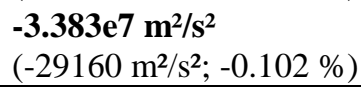 \\
\hline 3a) & $\begin{array}{l}\text { Ascent Anomaly: } \\
\text { Reduced Isp }\end{array}$ & $\begin{array}{l}\mathbf{7 9 . 3 5 8} \mathbf{k m} \\
(-32.463 \mathrm{~m} ;-0.041 \%)\end{array}$ & $\begin{array}{l}7.256 \mathbf{~ k m} / \mathbf{s} \\
(-4.178 \mathrm{~m} / \mathrm{s} ;-0.058 \%)\end{array}$ & 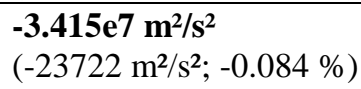 \\
\hline 3b) & $\begin{array}{l}\text { Ascent Anomaly: } \\
\text { Separation Time }\end{array}$ & $\begin{array}{l}\mathbf{7 8 . 5 5 3} \mathbf{~ k m} \\
(-35.086 \mathrm{~m} ;-0.045 \%)\end{array}$ & $\begin{array}{l}7.087 \mathbf{~ k m} / \mathbf{s} \\
(-4.001 \mathrm{~m} / \mathrm{s} ;-0.056 \%)\end{array}$ & $\begin{array}{l}\mathbf{- 3 . 5 3 6 e} 7 \mathbf{~ m}^{2} / \mathbf{s}^{2} \\
\left(-21790 \mathrm{~m}^{2} / \mathrm{s}^{2} ;-0.080 \%\right)\end{array}$ \\
\hline 3c) & $\begin{array}{l}\text { Ascent Anomaly: } \\
\text { Failure at Lift-Off }\end{array}$ & $\begin{array}{l}\mathbf{6 9 . 7 9 3} \mathbf{~ k m} \\
(-43.410 \mathrm{~m} ;-0.062 \%)\end{array}$ & $\begin{array}{l}7.098 \mathrm{~km} / \mathrm{s} \\
(-11.477 \mathrm{~m} / \mathrm{s} ;-0.162 \%)\end{array}$ & 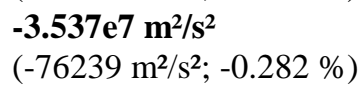 \\
\hline 3d) & $\begin{array}{l}\text { Ascent Anomaly: } \\
\text { Failure at Max-Q }\end{array}$ & $\begin{array}{l}\mathbf{7 3 . 4 6 6 ~ k m} \\
(-45.204 \mathrm{~m} ;-0.062 \%)\end{array}$ & $\begin{array}{l}7.290 \mathrm{~km} / \mathrm{s} \\
(-8.685 \mathrm{~m} / \mathrm{s} ;-0.119 \%)\end{array}$ & 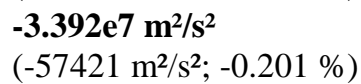 \\
\hline
\end{tabular}


Significantly higher performance reductions can be noticed when comparing the investigated anomaly scenarios with the nominal ascent trajectory. The highest loss occurs in case of an engine failure at lift-off which reduces the specific orbital energy at MECO by $-5.8 \%$ compared to the nominal case. Based on the current state of the simulation study these deficits could probably be compensated by adapted descent trajectories in order to fulfil the mission. However, further detailed investigations of the combined ascent and descent trajectory are necessary to evaluate the criticality of the anomaly scenarios for the mission's success.

\subsection{Feasibility of the TVC System}

An important aspect for dimensioning of the TVC actuators and the feasibility assessment of the control system are the maximum required TVC deflection angles during ascent flight. The necessary deflections of the SpaceLiner Main Engines are shown in FIG 18 in case of a moderately disturbed atmosphere. As can be seen the maximum vertical engine deflections are limited to $\pm 2.5^{\circ}$ in all flight conditions, while the lateral deflection angles remain below $\pm 1.4^{\circ}$. Compared to the gimbal limit of $\pm 8.5^{\circ}$ of typical liquid rocket engines [27] these deflections can be considered acceptable. It can also be noticed that the successive throttling of the SpaceLiner engines after $\mathrm{t}_{\mathrm{MET}}=150 \mathrm{~s}$ effectively limits the necessary vertical deflections of the TVC actuators. The yaw moment, which is induced by the asymmetric throttling of the engines, only provokes a small lateral deflection of all engines.
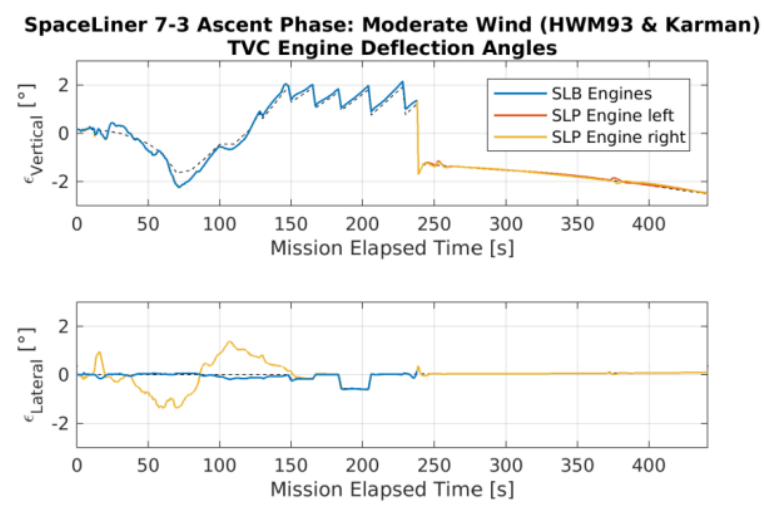

FIG 18. Lateral and vertical TVC deflections of the SpaceLiner Main Engines during ascent flight

When comparing the individual simulation cases it has been discovered that the engine deflections for roll control are sensitive to asymmetric loads as they occur e.g. during crosswinds or in case of an engine failure. An overview of the ranges of the deflection angles for the different scenarios is given in FIG 19. While for most engines the gimbal range remains almost constant under disturbances, the lateral deflection of the SpaceLiner Passenger Stages engines, which are providing roll control during the mated ascent phase, increase significantly under asymmetric loads. Providing sufficient roll control can therefore be identified as a limiting factor in the design of the TVC system. However, since all engine deflections are significantly below the predefined gimbal limit of $\pm 8^{\circ}$ for the SLMEs, no critical influence of the flight dynamics on the concept's feasibility can be identified based on the investigated simulation cases. Furthermore, as sufficient margins are present towards the gimbal limit of all engines, no necessity to incorporate aerodynamic actuators or the RCS system in the ascent control loop can be observed.

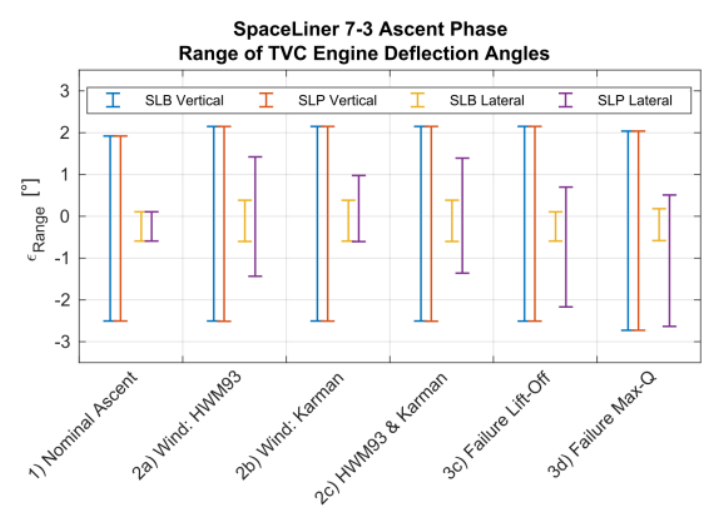

FIG 19. Ranges of the TVC deflections of the SpaceLiner Main Engines for the different simulation cases

\subsection{Trimmability and Aerodynamic Stability}

A necessary condition for the controllability of the SpaceLiner during ascent flight is the ability to trim the aerodynamic moments in all flight conditions with the TVC system. Concerning the nominal ascent trajectories FIG 20 visualizes the maximum TVC trim deflections for all flight conditions and variable angles of attack, and FIG 21 for variable sideslip angles respectively. Since all SpaceLiner Main Engines possess a gimbal limit of $\pm 8^{\circ}$ no moment trimming can be performed in the hatched flight states. Therefore at the point of maximum aerodynamic pressure the admissible flight envelope needs to be restricted to $-7^{\circ} \leq \alpha_{\text {Max-Q }} \leq 4^{\circ}$ and $-3^{\circ} \leq \beta_{\text {Max- }}$ $\mathrm{Q} \leq 3^{\circ}$. Since for all nominal and off-nominal simulation cases the aerodynamic angles have been limited in the Max-Q regime at $t_{\text {MET }}=50-100 \mathrm{~s}$ to $-0.1^{\circ} \leq \alpha \leq 1^{\circ}$ and $|\beta| \leq 0.7^{\circ}$, this envelope includes sufficient margins for the current mission design.

Generally, the trimmability of the aerodynamic moments can be ensured for all flight conditions up to lateral aerodynamic pressures of $\mathrm{q} \alpha \leq 2000 \mathrm{~Pa} \operatorname{rad}$ and $\mathrm{q} \beta \leq$ $1500 \mathrm{~Pa}$ rad. This restriction has only a marginal impact on the admissible flight envelope of the SpaceLiner since lateral aerodynamic pressures in this order of magnitude are already not desirable due to the occurring structural loads.

Another relevant control characteristic of the SpaceLiner is the aerodynamic stability. In contrast to the trimmability the aerodynamic stability is not a necessary condition for 
the general controllability of the vehicle, since the system's stability can also be provided by the flight control system. Especially in upper atmospheric layers above $50 \mathrm{~km}$ the influence of the aerodynamic pressure on the vehicle dynamics is almost negligible as already shown in chapter 4.1. Here, the aerodynamic stability has practically no implications on the stability of the entire system. Nevertheless, in lower altitudes aerodynamic stable flight conditions are desirable to allow for a simple flight control system.

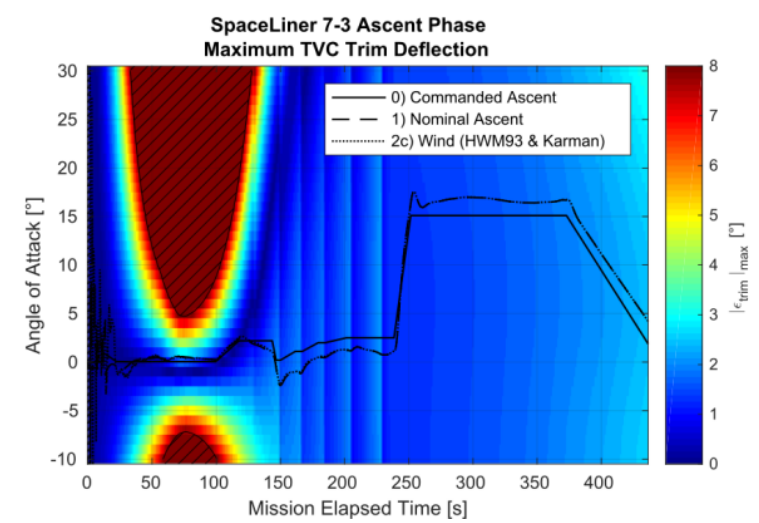

FIG 20. Maximum TVC trim deflections of the SpaceLiner Main Engines for variable angles of attack; Non-trimmable flight conditions are hatched

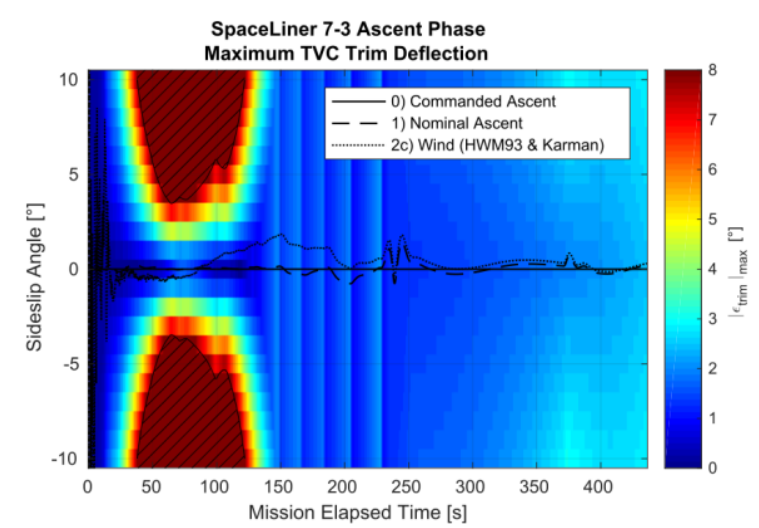

FIG 21. Maximum TVC trim deflections of the SpaceLiner Main Engines for variable sideslip angles; Non-trimmable flight conditions are hatched

The longitudinal aerodynamic stability derivative $c_{m \alpha}$ is shown in FIG 22 for a nominal ascent trajectory, and the lateral stability derivative $c_{n \beta}$ in FIG 23 respectively. In the contour plot all instable flight conditions are hatched, while a dashed line indicates the practical limit for the influence of aerodynamic effects. It should be noticed that lateral and longitudinal aerodynamic stability is given for the first flight phases except for very large angles of attack, where lateral stability cannot be ensured. In higher altitudes the SpaceLiner passes through aerodynamic instable flight states, but these regimes can be disregarded in the assessment of the system's controllability due to the lack of sufficient aerodynamic pressure.

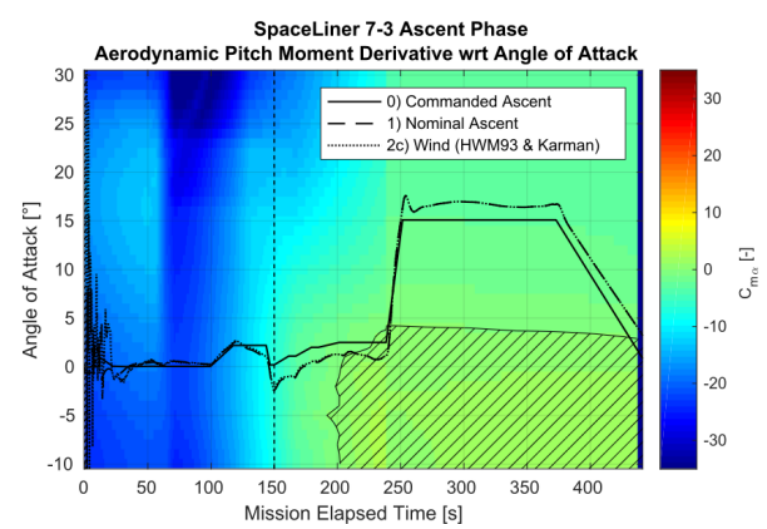

FIG 22. Longitudinal aerodynamic stability derivative $c_{m \alpha}$ for off-nominal angles of attack; Aerodynamic instable flight conditions are hatched

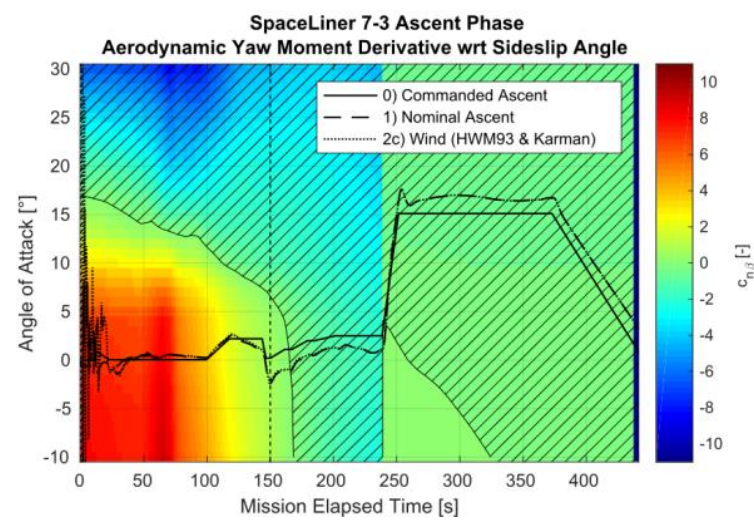

FIG 23. Lateral aerodynamic stability derivative $c_{n \beta}$ for off-nominal angles of attack; Aerodynamic instable flight conditions are hatched

\section{CONCLUSIONS AND OUTLOOK}

In this simulation study a comparison of the rigid-body vehicle dynamics with idealized ascent trajectories has been realized. It has been observed that small performance losses arise from the rotational motion of the SpaceLiner, but in relation to the complete ascent flight these deviations are marginal and can be covered by the system's design margins. The idealized reference trajectories could be tracked by the SpaceLiner Vehicle model with a sufficient positioning accuracy. Generally, the deviation was of a similar order of magnitude as the vehicle's dimensions. For the most critical flight phase during lift-off an improved accuracy below $4.5 \mathrm{~m}$ could be achieved. These conclusions stay true for the nominal as well as the investigated off-nominal ascent trajectories. Therefore the influence of the rotational degrees of freedom on the mission's design can generally be stated as secondary to the translational ascent flight dynamics.

Based on the simulation results detailed investigations of the controllability of the SpaceLiner have been undertaken. It could be shown that the TVC system of the SpaceLiner is sufficiently dimensioned to counteract asymmetric thrust settings and atmospheric disturbances during ascent flight. Adequate design margins to the 
mechanical gimbal limit of the SLMEs could be shown for all simulation cases. Generally, it could be noticed that providing sufficient roll control will be the limiting design case for the TVC system. The trimmablility of the vehicle has been ensured for all nominal and many off-nominal flight conditions, only limiting the achievable flight envelope at Max-Q to acceptably small aerodynamic angles. The aerodynamic stability of the SpaceLiner could be shown for all flight states with significant aerodynamic pressure. In higher altitudes aerodynamically instable flight conditions could be observed, but their influence on the vehicle dynamics can be considered negligible compared to the dominant TVC system. Finally, it can be stated that the SpaceLiner Vehicle is sufficiently controllable during ascent flight by its TVC system and the implemented preliminary flight controller.

Regarding the vehicle's ascent flight dynamics for the investigated flight scenarios, generally no evidence has been found which would contradict the feasibility of the SpaceLiner concept. Nevertheless further investigations of critical mission scenarios should be undertaken. This would include ascent trajectories with very strong environmental disturbances as well as combined analyses of ascent and descent trajectories, especially in case of operational anomalies. Based on this data an operational flight envelope could be defined for the SpaceLiner Vehicle to finally assess the concept's feasibility.

Furthermore, some simplifications have been made in the analyzed SpaceLiner model. Elastic effects of the vehicle's structure as well as fuel sloshing have been neglected by the rigid-body assumption, even though these effects might have an influence on the ascent flight dynamics. Their significance should be investigated in continuative studies. Also the influence of the preliminary designed flight controller on the system's dynamics has not been analyzed by this study. Here, a comparative study of different rapid control prototyping algorithms is currently being conducted.

\section{REFERENCES}

[1] Sippel, M., Klevanski, J., Steelant, J.: Comperative Study on Options for High-Speed Intercontinental Passenger Transports: Air-Breathing- vs. RocketPropelled. IAC-05-D2.4.09, 56th International Astronautical Congress, Fuduoka (2005)

[2] Sippel, M., Foreest, A.: SpaceLiner Rocket-Powered High-Speed Passenger Transportation Concept Evolving in FAST20XX. IAC-10-D2.4.06, 61st International Astronautical Congress, Prague (2010)

[3] Sippel, M., Schwanekamp, T., Trivailo, O., Kopp, A., Bauer, C., Garbers, N.: SpaceLiner Technical Progress and Mission Definition. AIAA 2015-3582, 20th AIAA International Space Planes and Hypersonic Systems and Technologies Conference, Glasgow, 2015
[4] Sippel, M., Schwanekamp, T., Ortelt, M.: Staged Combustion Cycle Rocket Engine Subsystem Definition for Future Advanced Passenger Transport. Space Propulsion, No. 30, Cologne (2014)

[5] Schwanekamp, T., Ludwig, C., Sippel, M.: Cryogenic Propellant Tank and Feedline Design Studies in the Framework of the CHATT Project. AIAA 20142370, 19th AIAA International Space Planes and Hypersonic Systems and Technologies Conference, Atlanta (2014)

[6] Valluchi, C., Sippel, M.: Hypersonic Morphing for the SpaceLiner Cabin Escape System. 7th European Conference for Aeronautics and Space Science EUCASS, Milan (2017)

[7] Sippel, M., Schwanekamp, T., Bauer, C., Garbers, N., Foreest, A. van, Tengzelius, U., Lentsch, A.: Technical Maturation of the SpaceLiner Concept. AIAA 2012-5850, 18th AIAA International Space Planes and Hypersonic Systems and Technologies Conference, Tours (2012)

[8] Sippel, M., Bussler, L., Kopp, A., Krummen, S., Valluchi, C., Wilken, J., Prévereaud, Y., Vérant, J.L., Laroche, E., Sourgen, F., Bonetti, D.: Advanced Simulations of Reusable Hypersonic Rocket-Powered Stages. AIAA 2017-2170, 21st AIAA International Space Planes and Hypersonic Systems and Technologies Conference, Xiamen (2017)

[9] Sippel, M., Trivailo, O., Bussler, L., Lipp, S., Valluchi, C., Kaltenhäuser, S., Molina, R.: Evolution of the SpaceLiner towards a Reusable TSTOLauncher. IAC-16-D2.4.03, 67th International Astronautical Congress, Guadalajara (2016)

[10] Krummen, S.: Investigation of flight dynamics and effects of rotational degrees of freedom on the flight performance of asymmetric space transportation systems based on the SpaceLiner 7 concept. SART TN-010/2016, Bremen (2016)

[11] The MathWorks Inc.: Product Documentation. https://www.mathworks.com/help/index.html (2018). Accessed 16 February 2018

[12] Wilken, J., Bussler, L.: SpaceLiner System Specification Document, SL-SS-SART-00026-1/0, Bremen (2017)

[13] Schwanekamp, T., Morsa, L., Zuppardi, G., Molina, R.: SpaceLiner 7-2 Aerodynamic Reference Database. SART TN-026/2012, Bremen (2012)

[14] Rosema, C., Doyle, J., Auman, L., Underwood, M., Blake, W.: Missle Datcom: User's Manual - 2011 Revision. AFRL-RB-WP-TR-2011-3071, Ohio (2011)

[15] Casali, E., Bussler, L., Sippel, M.: Investigation of feasible flight trajectories and re-entry atmospheric guidance for SpaceLiner 7. SART TN-014/2015, Bremen (2015)

[16] Brockhaus, R., Alles, W., Luckner, R.: Flugregelung. 3rd edn., Springer, Heidelberg (2011)

[17] Aström, K., Hägglund, T.: Automatic Tuning of Simple Regulators with Specification on Phase and 
Amplitude Margins. Automatica, Vol. 20, No. 5, 645651 (1984)

[18] Yu, C.-C.: Autotuning of PID Controllers. A Relay Feedback Approach. 2nd edn., Springer, Heidelberg (2006)

[19] Wilson, D.I.: Relay-based PID tuning. Automation and Control, Vol. 2005, 10-11 (2005)

[20]Zipfel, P.H.: Modeling and Simulation of Aerospace Vehicle Dynamics. American Institute of Aeronautics and Astronautics, Reston (2007)

[21] U.S. Department of Defense: World Geodetic System 1984, NIMA TR8350.2, 3rd edn (2000)

[22] National Aeronautics and Space Administration: U.S. Standard Atmosphere 1976. NASA-TM-X-74335, Washington (1976)

[23] Hedin, A.E., Fleming, E.L., Manson, A.H., Scmidlin, F.J., Avery, S.K., Clark, R.R., Franke, S.J., Fraser, G.J., Tsunda, T., Vial, F., Vincent, R.A.: Empirical Wind Model for the Upper, Middle and Lower Atmosphere. Journal of Atmospheric and Terrestrial Physics, Vol. 58, No. 13, 1421-1447 (1996)

[24] U.S. Department of Defense: Flying Qualities of Piloted Aircraft. MIL-HDBK-1797 (1997)

[25] Trivailo, O., Sippel, M., Valluchi, C., Lipp, S., Bussler, L., Kopp, A., David, E.: SpaceLiner Mission Requirements Document. SL-MR-SART-00001-1/2, SART TM-005/2016, Bremen (2016)

[26] Casali, E., Bussler, L., Sippel, M.: Investigation of feasible flight trajectories and re-entry atmospheric guidance for SpaceLiner 7. SART TN-014/2015, Bremen (2015)

[27] Dumoulin, J.: NSTS 1988 News Reference Manual. http://science.ksc.nasa.gov/shuttle/technology/stsnewsref/stsref-toc.html\#sts-mps (2000). Accessed 20 August 2017 